\title{
Haemorrhagic suprarenal pseudocysts: a systematic review of a rare condition from family physicians' perspective during the COVID-19 global pandemic
}

\author{
Adekunle Olowu'*, Adel Abbas Alzehairy² \\ 'Consultant Family Medicine, Al Thumama Health Centre, Primary Health Care Corporation, Doha, Qatar \\ ${ }^{2}$ Formerly Specialist Family Medicine, Al Thumama Health Centre, Primary Health Care Corporation, Doha, Qatar
}

\section{Article Info}

\section{Article Notes}

Received: March 07, 2021

Accepted: May 10, 2021

\section{*Correspondence:}

${ }^{*}$ Dr. Adekunle Olowu, Consultant Family Medicine, Al

Thumama Health Centre, Primary Health Care Corporation, Doha, Qatar; Email: kunletinu@hotmail.com.

${ }^{\circ} 2021$ Olowu A. This article is distributed under the terms of the Creative Commons Attribution 4.0 International License.

\section{Keywords:}

Adrenal cysts

Family physician

General practitioners

Haemorrhagic cysts

Huge

Perspectives

Primary care centre

Pseudocyst

Suprarenal cyst

Unusual presentation
Abstract

Adrenal cysts are rare lesions that could be epithelial, endothelial, parasitic or haemorrhagic ${ }^{1}$, as well as pseudocysts. Haemorrhagic adrenal cysts are extremely rare and are often asymptomatic, so diagnosis can be really challenging. This can prove really difficult for primary care physicians who are often the frontline clinicians these patients tend to present to. They are usually benign lesions and do not often cause mortality if detected early and prompt surgery is done, as was the case with the patient in our case report ${ }^{4}$. When they do become symptomatic, they can present with different systemic symptoms as documented in literature, including in our case report ${ }^{2,4}$. Diagnosis is usually through Ultrasound and CT Scan and management is largely laparoscopic or open excision depending on the size of the lesion, surgical expertise and local protocol. Most patients make full recovery and mortality is extremely low ${ }^{3}$. The aim of this review is to provide a broader overview of the subject, highlight salient points in several studies relating to haemorrhagic cysts, provide an up to date follow up information on the index patient in our case report and to explore possible areas for future study ${ }^{4,6}$. This review also includes a suggested management algorithm and intends to emphasize the fact that patients who present in primary, urgent or emergency care settings with persistent nonspecific symptoms should be investigated for rare diseases.

\section{Introduction}

In the light of the current COVID-19 pandemic that has not only stretched the resilience and capacities of global health services but also changed the way we work, one of the greatest challenges that physicians-primary care physicians, general practitioners, endocrinologists-all over the world face is making the right diagnostic decisions for their patients, especially those who present with recurrent non-specific symptoms. This systematic review is an attempt not only to highlight in depth the key features of the subject topic of haemorrhagic adrenal cysts and re-emphasize the need for appropriate investigation of patients with recurrent symptoms, but also to provide updates on our index patient who made a full recovery ${ }^{4}$, as well as to indicate an important opportunity for a future study on which the authors are currently working. Hopefully this systematic review, with the help of the suggested management algorithm, will highlight a few points that will enhance your clinical approach to patient care and help achieve an improvement in clinical outcomes in your areas of practice. Adrenal cystic lesions are rare and are often pseudocysts. Diagnosis is often a clinical challenge especially for primary care physicians who often have to detect, diagnose and refer them early to prevent complications and reduce 
mortality ${ }^{1}$. Adrenal pseudocysts are benign cystic masses that originate from the adrenal cortex or medulla and are confined within a fibrous wall. There are four classes of adrenal gland cysts: epithelial, endothelial, and parasitic and pseudocysts but they can also become haemorrhagic ${ }^{2}$. A review of 8 cases in a study in the United States revealed lesion sizes ranging from $1.8-10 \mathrm{~cm}$, though the size of the lesion does not correlate with symptoms, but in our case report the lesion was larger ${ }^{3}$. They are often seen in the 5 th and the 6th decades of life, but can also be diagnosed during pregnancy ${ }^{13,14,16}$. Haemorrhagic adrenal cysts are even rarer. They are relatively uncommon and rarely present with symptoms. However, they can cause symptoms through repeated episodes of trauma, infection or bleeding ${ }^{2}$. When they do, they are often non-specific symptoms, for example respiratory symptoms as highlighted in our case study, left testicular discomfort as identified by a case report in the United Kingdom ${ }^{8}$ and epigastric pain and abdominal distension ${ }^{15}$. An Indian case report even reported unusual presentations of hypertensive retinopathy, virilization and bleeding diathesis ${ }^{17}$.

\section{Literature Review}

Review Methodology: An in-depth and detailed online search of the literature was done which included resources like PubMed, ClinicalKey, AccessMedicine, Web of Science, Google Scholar, ProQuest Research Library, ScienceDirect, DynaMed, UpToDate, SciVal and BrowZine. Search terms used included "adrenal cysts" "adrenal pseudocysts" "suprarenal cysts" "haemorrhagic cysts" and "haemorrhagic pseudocysts". The searches identified over 30 articles with several interesting findings. Inclusion criteria were articles involving case reports or case reviews of the subject topic with clarity of diagnosis and treatment methods written in English and published within the last 5 years (2016-2021). Those not meeting these criteria were excluded, including cases reported in children, pregnancy or diagnosed prenatally, leaving only about 4 articles whose interesting findings are summarized as in our systematic review (Table 1). In total 26 cases were reviewed, with a mean age of $38.75 y e a r s$, preponderance of females(15/26[57.7\%]), but equal right and left-sided lesion split (13/26[50\%]). $18(69 \%)$ of cases were incidental findings while $2(7.7 \%)$ were malignant.
Haemorrhagic adrenal cysts are rarely symptomatic and often found incidentally during surgery for other conditions or at autopsy, except when they are large as in the case of a giant adrenal pseudocyst which was surgically removed $^{1,3}$. The search on pseudocysts also revealed only a few cases documented over several years, often detected at pathological examinations. Treatment is by surgical excision which can be done laparoscopically depending on the size of the lesion ${ }^{1,4,6,7,10}$. A 20-year review of cystic adrenal lesions at an academic medical centre revealed only 12 pseudocysts out of 31 cysts, out of a total of 4500 adrenal gland specimens reviewed ${ }^{2}$.

A clinicopathologic analysis of 55 cases of adrenal lesions revealed they were mostly right sided with a female predominance, though our index patient is male and had a left sided lesion. They also discovered that they are often associated with hormonal hypersecretions and endocrinological abnormalities ${ }^{3,4}$. Intracystic haemorrhage is one of the complications of adrenal pseudocysts, which our patient had ${ }^{4}$. Haemorrhagic complication of adrenal cysts is usually associated with factors like coagulopathies, Von Willebrand's disease, thrombocytopenia, antiphospholipid syndrome, previous therapy with clopidogrel or steroids and rupture of a prior tumor ${ }^{18}$, none of which our patient had. The diagnosis of adrenal cysts or pseudocysts is largely by imaging (Ultrasound Scan, CT Scan and MRI Scan) as confirmed in a review of 6 patients over 3 years ${ }^{5}$, while the use of laparoscopy, as enumerated by another study, is important not only in diagnosis but also in management of symptomatic cases ${ }^{7}$. They further clarified and concluded that laparoscopic decortication and marsupialization can be the preferred option for treatment for symptomatic smaller cysts, while the larger ones can be managed by laparoscopic partial to total adrenalectomy ${ }^{7}$. However, laparotomy was required in our case report patient due to the size of his lesion ${ }^{4}$. Management of adrenal or suprarenal cysts is largely by surgical excision to exclude malignancy, as enumerated in an American study, where they reviewed 613 cases, including those they had managed, where they found that only $7 \%$ of their cases were malignant or potentially malignant, with only 1 reported malignancy, and concluded that aspiration of the cysts may be done ${ }^{6}$. Several other diagnoses to keep

Table 1: Showing some characteristics of the studies included in our review

\begin{tabular}{|c|c|c|c|c|c|c|c|c|c|c|c|c|c|}
\hline \multirow[t]{2}{*}{ No. } & \multirow[t]{2}{*}{ Author } & \multirow[t]{2}{*}{ YR. } & \multirow[t]{2}{*}{ Cases } & \multirow[t]{2}{*}{$\begin{array}{c}\text { Age } \\
\text { (YRS) }\end{array}$} & \multicolumn{2}{|c|}{ Sex } & \multirow[t]{2}{*}{$\begin{array}{c}\text { Haem. } \\
\text { Pseudo-Cyst }\end{array}$} & \multirow[t]{2}{*}{$\begin{array}{c}\text { Incidental } \\
\text { Finding }\end{array}$} & \multirow[t]{2}{*}{ Malignancy } & \multirow[t]{2}{*}{ Mean Size(cm) } & \multirow[t]{2}{*}{ Treatment } & \multicolumn{2}{|c|}{ Side } \\
\hline & & & & & M & $\mathrm{F}$ & & & & & & Right & Left \\
\hline 1 & Geleit et al. & 2016 & 1 & 33 & 1 & Nil & Yes & No & Nil & Nil & Surgery & 0 & 1 \\
\hline 2 & Paramythiotis et al. & 2017 & 1 & 25 & Nil & 1 & Yes & No & Nil & $11.7 \times 9.3 \times 6.6$ & Surgery & 0 & 1 \\
\hline 3 & Zheng et al. & 2018 & 23 & 49 & 9 & 14 & Yes & 18 & 2 & 6.5 & Mainly Surgical & 13 & 10 \\
\hline \multirow[t]{2}{*}{4} & Olowu et al. & 2021 & 1 & 48 & 1 & Nil & Yes & No & Nil & $14.2 \times 13.5 \times 13.1$ & Surgery & 0 & 1 \\
\hline & Total & & 26 & 38.75 & $11[42.3 \%]$ & $15[57.7 \%]$ & & $18[69.9 \%]$ & $2[7.7 \%]$ & & & $13[50 \%]$ & $13[50 \%]$ \\
\hline
\end{tabular}


in mind when assessing these patients are benign and malignant lesions like adrenal or suprarenal masses e.g., adenocarcinomas or pheochromocytomas ${ }^{9}$. Other rare lesions identified in case reviews include adrenal angiolipoma as possible differential diagnosis ${ }^{17}$, as well as a mucinous cystic neoplasm ${ }^{11}$, and an unusual prenatal diagnosis of an adrenal cyst in pregnancy which had to be excised at laparotomy while the pregnancy was carried to term delivery ${ }^{12,13,16}$. Possibility of the diagnosis of congenital adrenal teratomas should also be borne in mind when examining neonates ${ }^{14}$, as well as a congenital adrenal cystic neuroblastoma ${ }^{16}$. There was even a unique case of a prenatally detected cystic adrenal mass associated with Beckwith-Wiedemann Syndrome ${ }^{19}$. There was no case report of a haemorrhagic suprarenal cysts presenting with predominantly respiratory symptoms of cough and shortness of breath in the literature, as our patient had. Adrenal cystic lesions are very rare with an incidence of about $0.064-0.18 \%$ as reported in pathologic series $^{20}$.

\section{Case Report Patient Updates}

The patient described in our original case report, a 48-year-old man, presented with predominantly respiratory symptoms [Please check the full Case Report in the authors' previously published article ${ }^{4}$. Abdominal Computerized Tomography Scan showed a left suprarenal cyst measuring $14.2 \times 13.5 \times 13.1 \mathrm{~cm}$, but he had a successful surgery which relieved his symptoms ${ }^{4}$. Post operatively the patient was well and all respiratory symptoms resolved apart from a mild episode of back pain 3 weeks post-op which eventually resolved spontaneously. However, three and a half months after surgery he developed a microcytic hypochromic anaemia for which he had Intravenous Iron Saccharate $100 \mathrm{mg}$ twice followed by oral ferrous fumarate $200 \mathrm{mg}$ twice a day, after which his haemoglobin normalized. He has been generally well since. There was no specific paper addressing post-op complication of iron deficiency anaemia in laparotomic excision of suprarenal pseudocysts but this is almost certainly linked to blood loss during surgery.

\section{Suggested Management Algorithm for Rare Adrenal or Suprarenal Lesions}

Based on the authors' experiences and this review article we would like to suggest the following Algorithm as a guide to the management of rare suprarenal or adrenal lesions. This is by no means conclusive and each patient should be managed by their physicians or surgeons as best as they can based on the individual patient's clinical features, circumstances and availability of services.

\section{Patient with Symptoms \& Signs suggestive of Adrenal Lesion/Cysts/Mass}

(Abdominal Pain \& tenderness, Abdominal Swelling, Shortness of breath and Back pain OR recurrent nonspecific respiratory or abdominal symptoms in multiple visits etc.)

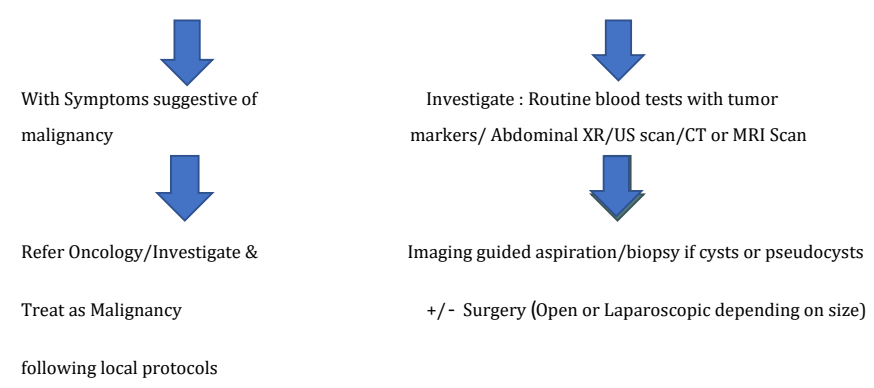

\section{Conclusion}

This review highlights important aspects of the literature about haemorrhagic adrenal cysts, providing a systematic review and suggesting a simple management algorithm that will help the average family physician or general practitioner to detect them promptly and refer on time for surgical intervention, especially patients who present with non-specific and recurrent symptoms. The authors are currently working on a 5-year retrospective review of adrenal lesions in Primary Health Care Centres and treated in their local hospitals in Qatar to determine the pattern of patient presentation, diagnosis and treatment outcomes.

\section{Conflict of Interest}

The authors declare no conflict of interest on this review.

\section{Acknowledgement}

We are grateful for the support of the Health Centre Manager Dr. Hanadi Al Hail, Consultant Family Physician and the Research Department, Primary Health Care Corporation, Qatar.

\section{References}

1. Paramythiotis D, Bangeas P, Karakatsanis A, et al. Surgical Management of a Giant Adrenal Pseudocyst: A Case Report and review of literature in the last decade. Case Reports in Surgery. 2018. (Available at: https://doi.org/10.1155/2018/8473231)

2. Sebastiano C, Zhao X, Deng FM, et al. Cystic lesions of the adrenal gland: our experience over the last 20 years. Human pathology. 2013; 44(9): 1797-803.

3. Zheng W, Fung KM, Cheng L, et al. Benign vascular tumors, cysts, and pseudocysts of the adrenal gland: a contemporary multi-institutional clinicopathological analysis of 55 cases. Human Pathology. 2018; 82: 95-102. 
4. Olowu AA, Alzehairy AA. A huge haemorrhagic suprarenal pseudocyst: an unusual presentation of a rare condition. BMJ Case Reports. 2021; 14(2): e235158. (Available at: https//doi:10.1136/ bcr-2020-235158)

5. Tung GA, Pfister RC, Papanicolaou N, et al. Adrenal cyst: imaging and percutaneous aspiration. Radiology. 1989; 173(1): 107-110.

6. Neri LM, Nance FC. Management of adrenal cysts. The American Surgeon. 1999; 65(2): 151-163 (Available at: https://search. proquest.com)

7. Castillo OA, Litvak JP, Kerkebe M, et al. Laparoscopic management of symptomatic and large adrenal cysts. The Journal of Urology. 2005; 173(3): 915-917. (Available at: https://doi.org/10.10970/01. ju.0000152177.35204.70)

8. Geleit RJ, Bhardwaj R, Fish D, et al. A unique presentation of a complex haemorrhagic adrenal pseudo cyst. BMJ Case Reports. 2016. (Available at: http://dx.doi.org/10.1136/bcr-2016-216246)

9. Siekavizza JL, Bernardino ME, Samaan NA. Suprarenal mass and its differential diagnosis. Urology. 1981; 18(6):625-32.

10. Ujam AB, Peters CJ, Tadrous PJ, et al. Adrenal pseudocyst: Diagnosis and laparoscopic management - A case report. International Journal of Surgery. 2011; 2(8): 306-308.

11. Medeiros LJ, Lewandrowski KB, Vickery AL Jr. Adrenal pseudocyst: a clinical and pathologic study of eight cases. Hum Pathology. 1989; 20(7): 660-5.

12. Erikci VP, Uçan B, Arslan OA, et al. A prenatally detected adrenal cyst treated by adrenal-sparing surgery: case report and review of the literature. Annals of Paediatric Surgery. 2011; 7(4): p152-154.

13. Trauffer PM, Malee MP. Adrenal Pseudocyst in Pregnancy. A Case Report. The Journal of Reproductive Medicine. 1996; 41(3): 195-197.

14. Yasui Y, Kohno M, Shironomae T, et al. Retroperitoneoscopic resection of a congenital adrenal teratoma in an infant. Journal of Paediatric Surgery Case Reports. 2013; 1(11): 391-394.

15. Erem C, Celik F, Reis A, et al. Large Adrenal Psudocyst Presenting with Epigastric Distress and Abdominal Distension. Medical Principles and Practice. 2005; 14(4): 284-287. (Available at: http:// doi:10.1159/000085752)

16. Chen $\mathrm{CP}$, Chen $\mathrm{SH}$, Chuang $\mathrm{CY}$, et al. Clinical and perinatal sonographic features of congenital adrenal cystic neuroblastoma: a case report with review of literature. Ultrasound Obstetrics Gynecology. 1997; 10(1): 68-73.

17. Ramareddy RS, Alladi A. Adrenal Mass: Unusual presentation and outcome. Indian J Med Paediatr Oncol. 2017; 38(3): 256-26.

18. Carsote M, Ghemigian A, Terzea D, et al. Cystic adrenal lesions: focus on paediatric population (a review). Clujul Med. 2017; 90(1): 5-12. (Available at: http://doi:10.15386/cjmed-677)

19. Merrot T, Walz J, Anastasescu R, et al. Prenatally Detected Cystic Adrenal Mass Associated with Beckwith-Wiedemann Syndrome. Fetal Diagnosis Therapy. 2004; 19(6): 465-469.

20. Bellantone R, Ferrante A, Raffaelli M, et al. Adrenal cystic lesions: Report of 12 surgically treated cases and review of the literature. Journal of Endocrinological Investigation. 1998; 21(2): 109-114. 